\title{
GIS-based Geostatistics and Multi-Criteria Evaluation of Steel Casing Failure
}

\author{
CHEN Jing ${ }^{1, ~ a ~}{ }^{*}$ LIU Yiliang ${ }^{2, b}$ and ZHU Qingjie ${ }^{3, c}$ \\ ${ }^{1}$ Department of Civil Engineering, Wuxi City College of Vocational Technology, \\ Wuxi 214153, Jiangsu Province, China \\ ${ }^{2}$ Department of Civil Engineering, North China Institute of Aerospace Technology, \\ Langfang 065000, Hebei Province, China \\ ${ }^{3}$ School of Petroleum Engineering, Changzhou University, \\ Changzhou 213016, Jiangsu Province, China \\ ajeany0315@sohu.com, ${ }^{b}$ liuyiliang1987@163.com, 'ojzhu@cczu.edu.cn \\ ${ }^{*}$ Corresponding author
}

Keywords: Spatial dependency, Geostatistics, kriging interpolating, Predictive model, GIS.

Abstract. Geostatistics is a very important tool of surface analysis in GIS application. Firstly, spatial dependency that represents the similar extent of neighboring points is used to analyze spatial data values and their locations. Spatial variability is calculated to assess spatial data in terms of distance and direction. Then, calculating results is used to create new surface images of spatial data values with kriging interpolating. As an example application, four sample data sets of oil wells, such as injection pressure, times of injection, injection temperature, and total injection, are analyzed. Predictive model of steel casing failure is constructed with four surface images that represent influence factors. Finally, the predictive distribution of steel casing failure is worked out, and some advice is proposed.

\section{Introduction}

Surface analysis is one of the most important aspects of GIS analysis, and geostatistics is the core of surface analysis. Since the high flexibility, many surfaces can be worked out by geostatistical techniques for the same data set. Therefore, it is essential to understanding geostatistical techniques in order to produce reasonable surfaces that are representations of reality. Many tools are included in geostatistics for the nature exploration of a data set, and spatial variability is the basic tool and focus of geostatistical analysis. For a varying spatial data set, the basic rule is that the closer in spatial locations, more similar in values. This was calculated by spatial variability analysis in GIS through quantified measures of spatial variability and auto correlation. A statistical characterization of a spatial data set can be analyzed by spatial variability with their values and locations of sample points. Usually, spatial variability is combined with techniques for interpolating surfaces in surface analysis, such as ordinary kriging $^{[1,2]}$. The combination of GIS and geostatistics is the foundation of surface analysis in GIS application, and has been applied in safety evaluation and risk assessment ${ }^{[3-6]}$.

There are several influence factors in safety evaluation or risk assessment, which is called multi-criteria evaluation (MCE). Since the 20th century, GIS-based MCE develops rapidly, especially weighted combination methods. Weighted linear combination (WLC) is the traditional method of MCE, and ordered weighted averaging (OWA) is a relatively new one ${ }^{[7,8]}$. Quantifier guided aggregation for OWA operators was first given by Yager in 1996. In recent years, many progresses have been made on OWA method, and applied to many decision- making domains ${ }^{[9-11]}$.

Semivariogram is calculated to assess the spatial variability or spatial dependency in geostatistical analysis for sample point data. Four images of influence factors are obtained based on spatial variability and kriging interpolating. Predictive model of steel casing failure is constructed based on multi-criteria 
evaluation. Finally, the predictive distribution of steel casing failure is worked out, and some advice is proposed.

\section{MCE Predictive Model}

For any point $i$ in geographical space, there are $j$ factors. Reordering factors' values from maximum to minimum, $\mathrm{z}_{i j}$ is the $j$-th factor value of failure risk, the predictive model based MCE is,

$$
z_{i}=\sum_{j=1}^{n}\left(\frac{u_{j} v_{j}}{\sum_{j=1}^{n} u_{j} v_{j}}\right) z_{i j}
$$

In which, $u$ is criteria weights, and $v$ is order weights.

If $\mathrm{z}_{i j}$ is unknown, it is expressed as $\mathrm{z}\left(x_{0}\right)$, the value $\mathrm{z}\left(x_{0}\right)$ can be calculated from known neighbor points as follow,

$$
z\left(x_{0}\right)=\sum_{l=1}^{n} \lambda_{l} z\left(x_{l}\right) \quad l=1,2,3 \mathrm{~L} n
$$

In which, $\mathrm{z}\left(x_{0}\right)$ is value of unknown point; $\lambda_{l}$ is weight for $z\left(x_{l}\right) ; z\left(x_{l}\right)$ is value of the $l$-th known point $x_{l}$. For ordinary kriging interpolation, weights are calculated from,

$$
\sum_{l=1}^{n} \lambda_{l}=1 \quad l=1,2,3 \mathrm{~L} n
$$

And,

$$
\sum_{l=1}^{n} \gamma\left(x_{l}-x_{m}\right) \lambda_{l}+\mu=\gamma\left(x_{0}-x_{m}\right) \quad m=1,2,3 \mathrm{~L} n
$$

In which, $u$ is Lagrange parameters, $\gamma$ is the semivariogram. The semivariogram is a tool to describe spatial variability.

For a data pair $\left(x_{i}, x_{i+h}\right)$, their values are $z(x), z(x+h)$, and the distance of two data is $h$. The semivariogram is expressed as,

$$
\gamma(h)=\frac{1}{2 n} \sum_{i=1}^{n}\left[z\left(x_{i}\right)-z\left(x_{i}+h\right)\right]^{2}
$$

In which, $n$ is the number of pairs with distance $h$.

The semivariogram can be presented as a surface image and a directional image. In surface image, the average variability is shown in all directions, which is in different lags. The center point means a zero lag, and lags increase in all direction from origin toward the boundary of research area. Usually, many images are plotted in different lags and different directions, in order to explain the spatial character of sample data. Both isotropic and anisotropic model are included, but anisotropic model is used in most cases, since spatial variability is usually anisotropic. With kriging interpolation, value of any unknown spatial point calculated as basis of the directional semivariogram. The directional semivariogram is expressed as a mathematical function, and can be also shown as a smooth curve. There are four parameters to define the mathematical function, such as sills, nugget, anisotropies and ranges. Surface images that come from the kriging interpolation can be used in multi-criteria evaluation to evaluate the safety or risk in engineering.

As equation (1)shown, there are two types of weights, criteria and order weights. The basic method to calculate the order weights is rank order method. In this method, weights are calculated according to their rank ordering, and different weight is obtained in different location. It is expressed as, 


$$
v_{j}=\frac{n-r_{j}+1}{\sum_{=1}^{n}\left(n-r_{l}+1\right)} \quad(j, l=1,2, \mathrm{~L} n)
$$

In equation (1), $u$ is criteria weight. Criteria weights indicate relative importance between two factors, for the $j$-th factor, weights are the same in different locations. Analytical hierarchy program (AHP) is the most useful method, and comparison matrix is constructed in this method to calculate criteria weights. For example, comparison matrix A is expressed as $\left[a_{i j}\right]$, and criteria weights are calculated through standardization as follow,

$$
u_{j}=\frac{u_{j}}{\sum_{i=1}^{n} \overline{u_{i}}} \quad i, j=1,2, \mathrm{~L}, n
$$

$\overline{u_{i}}$ comes from the column sum as,

$$
\bar{u}_{i}=\sum_{j=1}^{n} \bar{a}_{i j} \quad i, j=1,2, \mathrm{~L}, n
$$

And it comes from row sum,

$$
\bar{a}_{i j}=\frac{a_{i j}}{\sum_{k=1}^{n} a_{k j}} \quad i, j, k=1,2, \mathrm{~L}, n
$$

\section{An Example Application}

As an example application, four influence factors are selected to evaluate the risk of steel casing failure. The four influence factors are steam injection pressure, times of steam injection, injection temperature and total steam injection. Sample data are obtained from 120 wells, and ten of them are shown as table 1 . The purpose is to create surface images based on spatial variability analysis.

Table 1 Values of sample data

\begin{tabular}{|c|l|l|l|l|l|l|l|l|l|}
\hline No. & $\begin{array}{l}\text { Injection } \\
\text { pressure }\end{array}$ & $\begin{array}{l}\text { Times of } \\
\text { injection }\end{array}$ & $\begin{array}{c}\text { Injection } \\
\text { temperature }\end{array}$ & $\begin{array}{l}\text { Total } \\
\text { injection }\end{array}$ & No. & $\begin{array}{l}\text { Injection } \\
\text { pressure }\end{array}$ & $\begin{array}{l}\text { Times of } \\
\text { injection }\end{array}$ & $\begin{array}{c}\text { Injection } \\
\text { temperature }\end{array}$ & $\begin{array}{l}\text { Total } \\
\text { injection }\end{array}$ \\
\hline 1 & 14.483 & 6 & 322.25 & 14603 & 61 & 12.67333 & 15 & 340.33 & 67398 \\
\hline 2 & 12.75 & 3 & 320.27 & 5808 & 62 & 12.55625 & 16 & 333.29 & 46725 \\
\hline 3 & 10.775 & 12 & 329.71 & 31960 & 63 & 11.6062 & 16 & 314 & 44697 \\
\hline 4 & 14.05 & 4 & 323.67 & 9871 & 64 & 12.06 & 15 & 303.22 & 44439 \\
\hline 5 & 11.9357 & 14 & 335.5 & 36989 & 65 & 12.403 & 23 & 314.13 & 58183 \\
\hline 6 & 10.87273 & 11 & 334.33 & 33810 & 66 & 12.3153 & 13 & 310.33 & 26964 \\
\hline 7 & 10.8076 & 13 & 338 & 40956 & 67 & 12.91 & 20 & 303.43 & 48521 \\
\hline 8 & 11.125 & 8 & 335.08 & 21005 & 68 & 17.5142 & 14 & 325.78 & 61542 \\
\hline 9 & 10.491 & 12 & 329.56 & 32978 & 69 & 10.35 & 14 & 309.93 & 36373 \\
\hline 10 & 11.55833 & 12 & 317.73 & 33495 & 70 & 11.775 & 8 & 312 & 19379 \\
\hline
\end{tabular}

Spatial variability of four sample data sets is analyzed, and four Kriging interpolating surfaces imagines are crested.

Spatial Dependency Analysis. Four surface images of spatial variability are created according to the data in 120 wells. For example, the semivariogram imagines of times of injection and injection pressure are shown in figure 1. In each pixel, the color represents the value of average approximation to the semivariances of data pairs. The lowest variability is representing as dark color, and the green color for the highest variability. In the bottom of the images, the direction of calculation and lag distance are displaced. The direction degrees are read clockwise starting from the North.

The spatial variability for times of injection is shown as figure 1(a). It was found that the lowest semivariogram is at the degree of $110^{\circ}$. Semivariogram increases most rapidly in the direction $10^{\circ}$. The degree of spatial dependency across distance in $10^{\circ}$ is greater than direction $110^{\circ}$, it implies that 
north-east direction is the most anisotropic direction and rapidly changes in samples data. The spatial variability of injection pressure is shown as figure 1(b), which is treated as the second influence factor. Its trend is similar with the first influence factor, but slowly in change. The third factor of injection temperature and the fourth factor of total injection are also calculated, but not discussed here. The spatial variability of the third factor is similar with the second factor, and the forth influence factor is similar with the first one. This reveals that injection pressure and injection temperature are significant correlation, so with steam injection times and total steam injection.

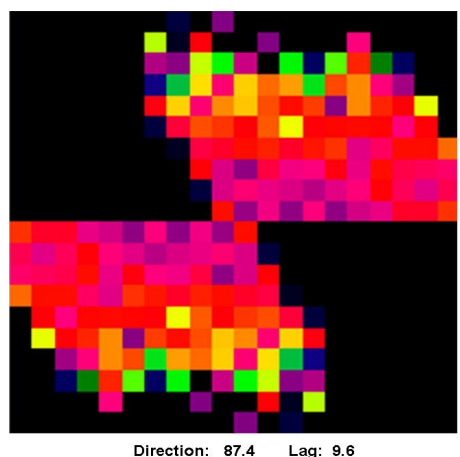

(a) Times of injection

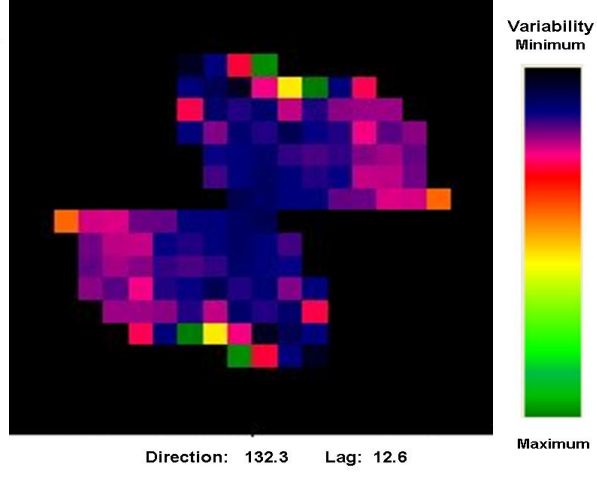

(b) Injection pressure

Fig. 1 Semivariogram imagines

Kriging Interpolating Surfaces. Interpolated surfaces are worked out through kriging method based on the equations (2) to (5). Those imagines are standardized from 0 to 255 , in which, the maximum risk is 255 , and 0 represents the minimum. The risk imagines come from times of injection and injection pressure are shown is shown in figure 2 .

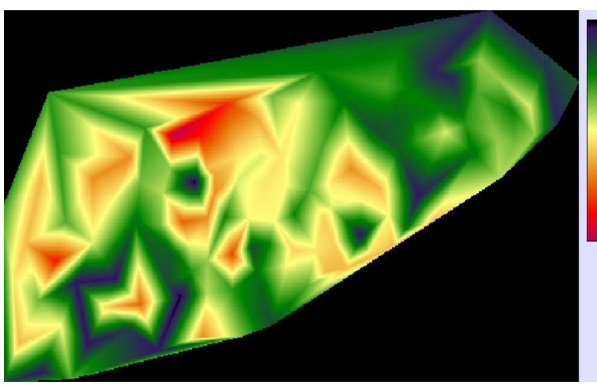

(a) Times of injection

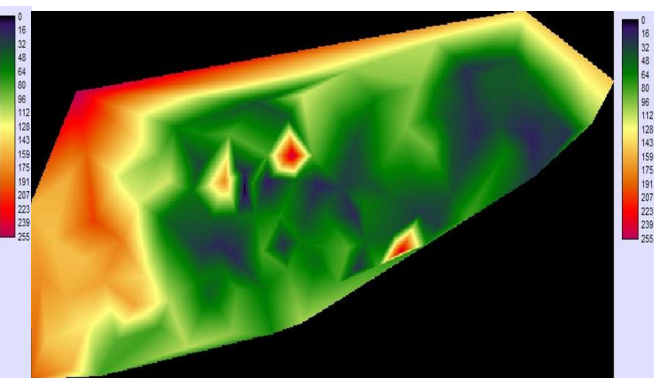

(b) Injection pressure

Fig. 2 Surface imagines

The steel casing failure risk can be investigated as basis of single surface imagines, but most important is to obtain the result of comprehensive evaluation.

Multi-Criteria Evaluation. Criterion weights and order weights are calculated according to equation (6) to (9). The results of criterion weights are $0.09,0.27,0.16$ and 0.48 . The results of order weights that come from equation (6) are $0.4,0.3,0.2,0.1$. Evaluation result is shown as figure 3.

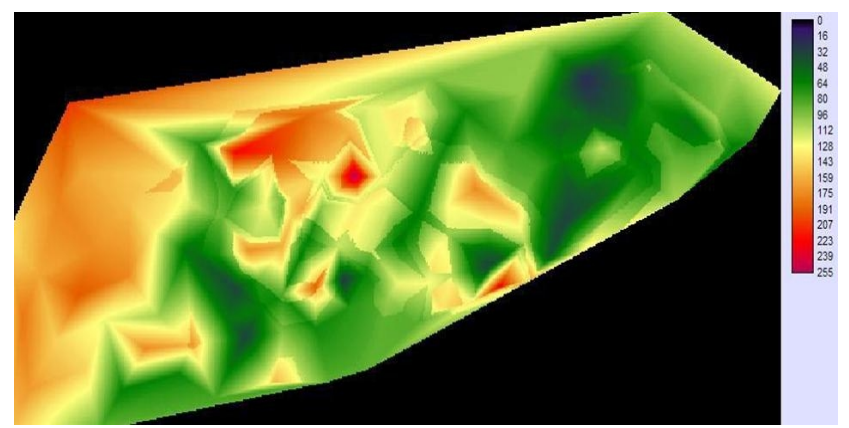

Fig. 3 Evaluation result 
The risk of steel casing failure (refer with fig.3) increases gradually from east to west. The safety areas locate in the eastern with properly values less than 100 . The most dangerous areas of steel casing failure locate in the northwest of the research area, and more attention should be paid. In order to reduce the risk of steel casing failure, some engineering measures should be taken to reduce the amount of steam injection. It is worth to discuss the influence of order weights. For example, order weights with $0.25,0.25,0.25$, and 0.25 , means equal order weights, will obtain the same result of weighted linear combination method. It means that weighted linear combination method is a special case of ordered weighted averaging method.

\section{Summary}

It is necessary to analyze the spatial variability is engineering risk assessment, which relative to distance and direction. Also, a great deal of time should be spent to model fitting with different directions and lags during semivariogram calculation. Interpolating surfaces imagines are created with kriging techniques, and MCE method is combined with spatial variability. This method is confirmed through an example application for risk assessment of steel casing failure.

\section{Acknowledgement}

This research was financially supported by the Twelfth Five Education Science Project of Jiangsu Province (D/2013/01/130), and Oversea Scholarship of Jiangsu Province (JS-2013-326).

\section{References}

[1] M.M. Gamez, L. Monrero, J.M. Orenzo, Kriging methodology for regional economic analysis, Intemational Advances in Eeonomic Researeh, 6(2000) 438-450.

[2] P.A. Burrough, GIS and geostatistics: Essential partners for spatial analysis, Environmental and Ecological Statistics, 8(2001)361-377.

[3] P. Pluch, Kriging and Splines: Theoretical Approach to Linking Spatial Prediction Methods, Jürgen Pilz (Eds), Interfacing Geostatistics and GIS, Springer Berlin Heidelberg, Berlin 2009, pp 45-56.

[4] D.C. Weidorf, Y. Zhu, Spatial variability of soil properties at Capulin Volcano, New Mexico, USA: Implications for sampling strategy, Pedosphere, 20(2010)185-197.

[5] C.H. Wang, J.S. Yuan, C.Q. Jiang, etal., Advance in Assessing of Heavy Metal Contamination in Soil using GIS and Geostatistics, Journal of Anhui Agri . Sci . , 40(2012)10414-10418.

[6] Q.J. Zhu, B. Hejmanowska, Analysis of GIS-based spatial variability and risk assessment, Journal of Chemical and Pharmaceutical Research, 5(2013)372-380.

[7] R.R. Yager, Using trapezoids for representing granular objects: applications to learning and OWA aggregation, Information Sciences, 178(2008)363-380.

[8] Y.J. Xu, Q.L. Da, Trapezoidal fuzzy ordered weighted averaging operator and its application to decision making, Journal of Southeast University (Natural Science Edition), 36(2006)1034-1038.

[9] Q.J. Zhu, Y. Su, D.D. Wu, Risk assessment of land-use suitability and application to Tangshan City, Int. J. Environment and Pollution, 42(2010)330-343.

[10] W.B. Xu, Q.J. Zhu, H.B. Jia, The Comprehensive Evaluation Method Based on GIS of Oil Well Casing Damage , Advanced Materials Research, 807-809(2013)2595-2601. 
[11] T.L. Saaty, J.S. Shang, An innovative orders-of-magnitude approach to AHP-based mutli-criteria decision making: Prioritizing divergent intangible humane acts, European Journal of Operational Research, 214(2011)703-715. 